\title{
La investigación en maíz en Pampas - Huancavelica : Avances y Perspectivas
}

Research in maize in Pampas - Huancavelica: Progress and Perspectives

\author{
${ }^{1,2}$ Pedro José García Mendoza ${ }^{\text {a }}$
}

\section{RESUMEN}

Desde el pasado mes de junio de 2018 la Universidad Nacional Autónoma de Tayacaja "Daniel Hernández Morillo" (UNAT), aprobó mediante resolución de la Comisión Organizadora el proyecto de maíz intitulado: "SELECCIÓN DE CULTIVARES Y CONSERVACIÓN DE LA IDENTIDAD GENÉTICA DE VARIEDADES DE MAÍZ (Zea mays L.) EN LA PROVINCIA DE TAYACAJA", el cual tiene como objetivo general seleccionar y desarrollar variedades de maíz con elevado potencial genético de producción y elevada calidad culinaria e industrial y establecer un programa de mantenimiento de la identidad genética de los cultivares seleccionados, que permita la producción de semilla de alta calidad, para mejorar la rentabilidad de toda la cadena agroproductiva vinculada al cultivo de maíz en la Provincia de Tayacaja. Para lograr este objetivo fue necesario realizar colectas de las principales variedades de maíz utilizadas por los productores agrícolas de la provincia de Tayacaja, con el propósito de efectuar evaluaciones de tipo agronómico, organolépticas y moleculares, las cuales permitirán identificar las variedades más promisorias para las diferentes regiones de la provincia. En la presente nota técnica se darán a conocer los avances logrados hasta el presente y los planes establecidos para los próximos dos años del proyecto.

\begin{abstract}
Since last June of 2018 the National Autonomous University of Tayacaja "Daniel Hernández Morillo" (UNAT), approved by resolution of the Organizing Committee the corn project entitled: "SELECTION OF CULTIVARS AND CONSERVATION OF THE GENETIC IDENTITY OF MAIZE VARIETIES (Zea mays L.) IN THE PROVINCE OF TAYACAJA ", whose general objective is to select and develop maize varieties with high genetic potential for production and high culinary and industrial quality and establish a program to maintain the genetic identity of the cultivars. selected, which allows the production of high quality seed, to improve the profitability of the entire agro-productive chain linked to the cultivation of corn in the Province of Tayacaja. To achieve this objective it was necessary to collect the main varieties of corn used by agricultural producers in the province of Tayacaja, with the purpose of making agronomic, organoleptic and molecular evaluations, which will allow identifying the most promising varieties for the different regions of the province. In the present technical note will be announced the progress made so far and the plans established for the next two years of the project.
\end{abstract}

'Universidad Nacional Autónoma de Tayacaja "Daniel Hernández Morillo".

${ }^{2}$ Instituto Nacional de Investigaciones Agrícolas (INIA), Venezuela. ${ }^{a}$ Ingeniero Agrónomo 


\section{INTRODUCCIÓN}

Una forma práctica y eficiente de mejorar los niveles de productividad de la provincia de Tayacaja, los cuales se ubican en alrededor de $1 / 3$ de la media nacional (MINAGRI, 2012), es identificando las variedades de maíz que presenten mayor potencial de producción, bien adaptadas a las condiciones agroecológicas de la región de la provincia de Tayacaja y con adecuada aceptación culinaria por el consumidor final del producto. Esto es factible de lograr a través de la evaluación de las poblaciones (variedades) de maíz utilizadas por los agricultores en la zona de estudio en diferentes ambientes de la región (Medina et al, 2002; Córdova, 1992).

Esta etapa se considera la más difícil y la que requiere de mayor inversión, debido a la necesidad de evaluar un gran número de genotipos en el mayor número de ambientes posibles (Souza et al, 2009). Posteriormente, se puede iniciar un programa de mejoramiento genético de las poblaciones, por medio de la selección recurrente, que permite incrementar, de manera gradual, la frecuencia de alelos favorables para los caracteres de importancia agronómica en el cultivo de maíz, manteniendo inalterable la variabilidad genética de la población, lo cual permite un mejoramiento genético progresivo de las variedades autóctonas y por ende de sus niveles de productividad (Paliwal, $2001 \mathrm{a}$; Hallauer, 1985). Adicionalmente, la implementación de un programa de mantenimiento varietal permite conservar la identidad genética de las variedades, garantizando la producción de semilla de elevada calidad genética (Paliwal, 2001b.

Para tomar las mejores decisiones en cada una de las etapas del proceso de selección de las variedades de superior comportamiento, se requiere no sólo del conocimiento sino también de la experiencia en muchas áreas de la genética vegetal, tales como la formación de poblaciones, manejo de germoplasma, estudios de comportamiento agronómico que permiten determinar la importancia o magnitud de los efectos de la interacción genotipo ambiente, uso y manejo de los efectos de la heterosis y la depresión por endocría en poblaciones de maíz tropical, entre otros, en donde la experiencia vivida en otras latitudes pueden brindar orientación efectiva para tomar las decisiones más acertadas, que permitan asegurar el logro de los objetivos planteados en el proyecto.

Por esta razón, el pasado mes de noviembre se recibió la visita de dos científicos del Centro Internacional de Mejoramiento de Maíz y Trigo (CIMMYT), especialistas en el área de mejoramiento genético, quienes estuvieron interactuando con el equipo responsable del proyecto y también lograron visitar algunas de las localidades donde se han establecido los experimentos. Durante las visitas a las regiones productoras de maíz de la provincia de Tayacaja, los investigadores 
del CIMMYT también interactuaron con los productores de maíz, quienes tuvieron oportunidad de exponerle los principales factores limitantes de la producción en sus respectivas zonas de producción.

Como producto de esta visita, se recibieron orientaciones en las áreas agronómicas y de mejoramiento genético del cultivo. En lo que respecta al área agronómica, se planteó la necesidad de realizar muestreos en el campo de los principales problemas fitopatológicos presentes en las zonas de producción, para ser analizadas en el laboratorio, a fin de determinar con exactitud los agentes causales de tales enfermedades y de esta manera poder idear alguna estrategia apropiada para su control. Adicionalmente, a través de los contactos de estos investigadores del CIMMYT se logró la adquisición de un equipo desarrollado por un investigador de la Universidad de Oklahoma, USA, para la siembra fertilizada de maíz y otros granos. Con esta misma Universidad, habrá oportunidad para lograr un entrenamiento del personal docente de la UNAT en algunas áreas de ingeniería agronómica e industrial.

En lo concerniente al área de mejoramiento genético del maíz, la prioridad observada por los científicos de CIMMYT es lograr la uniformidad de las variedades de maíz utilizadas por los agricultores, el establecimiento de una metodología apropiada para la producción de semilla de alta calidad física, genética y fisiológica y la realización de cruzamientos para la formación de un pool genético entre las diferentes variedades recolectadas, agrupadas en función de su uso (Cancha, Mote, etc.), para que sirvan como población base para inciar un programa de mejoramiento genético en el cultivo de maíz, con el cual la UNAT podrá poner a disposición de los productores agrícolas de la provincia y provincias vecinas nuevas variedades de maíz, bien adaptadas a las regiones productoras, con adecuada aceptación culinaria, pero con mayor potencial de producción que las variedades actuales. Serán nuevas variedades de maíz desarrolladas por la UNAT, a partir de las mismas variedades utilizadas por los productores de la región y con la participación activa de dichos productores. A este tipo de mejoramiento genético de cultivares se le llama mejoramiento genético participativo, donde los productores forman parte del equipo de trabajo que lidera la producción de los nuevos cultivares (ToledoMachado, et al., 2006; Rosas, 2001).

El referido proyecto de maíz de la UNAT contempla evaluaciones agronómicas, organolépticas y moleculares, cuyos resultados permitirán identificar las variedades más promisorias para las diferentes regiones de la provincia. Toda la información a recopilar en todos estas evaluaciones, requieren ser analizadas de la manera más apropiada, con el fin de aprovecharla en su totalidad, lo cual involucra no sólo el concurso de profesionales que conozcan a 
profundidad el manejo de datos univariados y multivariados, sino también de software apropiados, que sean amigables (en ambiente Windows y de fácil manipulación) y que se puedan adquirir en forma libre o a muy bajos costos.

Por esta razón, el proyecto también ha contemplado contactar a profesionales en el área de la estadísitica, a fin de que brinden asesoría técnica en este aspecto de las ciencias agrícolas. En este sentido, el pasado mes de noviembre se recibió también la visita de dos profesionales con amplia formación en el área de estadística, vinculada al área agronómica y de mejoramiento genético de plantas, quienes orientaron al personal responsable del proyecto respecto al tipo de análisis a realizar y la manera más apropiada para enfocar la propuesta, de manera de obtener resultados mucho más manejables y comprensibles desde el punto de vista biológico. Todo esto contribuirá a facilitar la toma de decisiones en el proyecto.

\section{MATERIALY METODOS}

Durante la asesoría, los profesionales en el área de estadística, propusieron el uso de diferentes software estadísticos que permiten hacer análisis de varianza de datos biométricos. No obstante, la mayoría adolecen de herramientas básicas para el manejo de análisis univariados y multivariados y algunos de los más completos, son muy costosos. Por esta razón se propuso el uso de algunas herramientas de Excel que permiten las programaciones con Macros para realizar la estadística descriptiva, que ayudan a detectar posibles errores en los datos, de tal forma que se puedan corregir en el momento y casi en línea estarían los Anavar con sus pruebas de medias.

También pusieron a disposición de la UNAT algunos paquetes estadísticos (Software), tales como el INFOSTAT, que es un programa para análisis estadístico de aplicación general desarrollado por la Universidad Nacional de Córdova (UNC), Argentina, bajo la plataforma Windows, el cual cubre tanto las necesidades elementales para la obtención de estadísticas descriptivas y gráficos para el análisis exploratorio, como métodos avanzados de modelación estadística y análisis multivariado. Una de las fortalezas es la sencillez de su interfaz combinada con capacidades profesionales para el análisis estadístico y el manejo de datos (Balzarini et al, 2008).

Debido al origen universitario, el programa tiene muchas facilidades para la enseñanza de la estadística que no son fáciles de encontrar en otros programas similares. Por otro lado, posee una versión en español muy valorada por los usuarios, especialmente por los estudiantes. Una propiedad casi única entre el software estadístico es la habilidad de InfoStat de conectarse con "R", una plataforma de desarrollo de algoritmos estadísticos de dominio público de gran crecimiento. $\mathrm{La}$ conexión con el programa "R" la hace de 
dos maneras: mediante un intérprete integrado que permite ejecutar script de " $\mathrm{R}$ " sin salir del ambiente de trabajo de InfoStat y mediante el desarrollo de aplicaciones que utilizan el motor de cálculo de "R", pero con la interfaz amigable que los usuarios esperan. De esta manera se pueden manejar modelos matemáticos complejos de manera más simples con la interfaz de InfoStat (Balzarini et al, 2008).

Adicionalmente, con esta asesoría también se logró obtener un software que sirve como una adecuada herramienta metodológica para la manipulación de todo el germoplasma recolectado en la provincia, el cual dará origen a las nuevas variedades de maíz producidas por la UNAT, así como también para el manejo de viveros y de ensayos de campo.

Algunos diseños de campo, tales como los Látices y Alfa-Látices no son fáciles de generar si no se cuenta con un software que haya sido desarrollado para ese propósito. Este Software, denominado "Fielbook" (Banziger y Barreto, 1999), fue desarrollado por el Centro Internacional de Mejoramiento de Maíz y Trigo (CIMMYT) y el mismo permite manejar pedigree, base de datos de bancos de germoplasmas, preparar diseños experimentales complejos y libros de campo de ensayos de investigación agrícola y otras áreas de las ciencias biológicas.

Hasta el presente, se plantaron experimentos en seis localidades de la provincia, donde se están evaluando 19 variedades de maíz recolectadas en la provincia, más seis variedades testigos proporcionadas por el INIA Cajamarca, para un total de 25 genotipos.

El objetivo de estos ensayos es evaluar el comportamiento agronómico de las variedades recolectadas y caracterizar dichas variedades desde el punto de vista morfológico, a fin de identificar las variedades más promisorias para las diferentes regiones de la provincia.

El diseño utilizado fue un Alfalátice $5 \times 5$ con tres repeticiones. La unidad experimental estuvo integrada por dos hileras de $4 \mathrm{~m}$ de longitud, utilizando arreglos espaciales de $0,80 \mathrm{~m}$ entre hileras y $0,20 \mathrm{~m}$ entre puntos de siembra. Se diseñó para colocar dos semillas por punto de siembra y posteriormente efectuar un raleo, dejando sólo una planta por punto de siembra.

Cada experimento tuvo una aleatorización distinta, generada de manera automática por el programa. Para cada ensayo se preparó un folder que contenía la lista de materiales probados (Tabla 1), información referente a las especificaciones del ensayo y del lugar donde se estableció el experimento, el protocolo para la toma de información del experimento, el mapa y libros de campo.

Las 19 variedades recolectadas en la provincia también están siendo evaluadas por su condición organoléptica en la Universidad Le Cordon Blue. Con base a la información recopilada en las evaluaciones agronómicas y las evaluaciones organolépticas se podrán 
se podrán definir las variedades que posean el mejor potencial para las diferentes regiones de la provincia de Tayacaja. Para el segundo año del proyecto se prevé la evaluación molecular de las variedades de maíz recolectadas en la provincia, lo cual permitirá una caracterización más precisa desde el punto de vista genético, información que será de gran utilidad para orientar al personal en la realización de los cruzamientos entre los materiales recolectados para formar los pool genéticos.

\section{PERSPECTIVAS DEL PROYECTO PARA LOS PRÓXIMOS AÑOS}

Para el segundo año del proyecto, también se prevé la realización de actividades de capacitación a nivel de las comunidades de la provincia, tanto en el área agronómica, como en lo que respecta a la producción de semilla.

Tabla 1. Lista de materiales experimentales y comerciales, lugar de origen y nombre dado a las variedades.

\begin{tabular}{|c|c|c|c|c|}
\hline Entrada & Distrito & Localidad & Productor & $\begin{array}{l}\text { Variedad } \\
\text { recolectada }\end{array}$ \\
\hline 1 & \multirow{4}{*}{$\begin{array}{l}\text { Daniel } \\
\text { Hernández }\end{array}$} & \multirow{2}{*}{ Los Ángeles de Cucharán } & Mauro Aguirre & Astilla Amarilla \\
\hline 2 & & & Alejandro Bobadilla & Astilla Blanca \\
\hline 3 & & Pata Pampa, Santa María & Jaime Salazar Concha & Carhuay \\
\hline 4 & & Rundo & Adalberto Ega & Carhuay \\
\hline 5 & \multirow{3}{*}{ Quishuar } & Anjara Pata & Karina Contreras & Carhuay \\
\hline 6 & & Pachas & María Grinalda Hidalgo Pérez & Bolón \\
\hline 7 & & Yacutoclla & Richard Vanza Vegas & Chulpy \\
\hline 8 & \multirow{4}{*}{ Pampas } & \multirow{2}{*}{ Ahuayta Casay } & Sergio Pérez Zurca & Astilla Blanca \\
\hline 9 & & & Gerardo Vilca Quispe & Astilla Blanca \\
\hline 10 & & Gentil Pampas & Porfirio Quispe & Cusqueado \\
\hline 11 & & Mantacra & Climaco Culcapuza Ilizarbes & Carhuay \\
\hline 12 & \multirow{2}{*}{ Colcabamba } & Agua Dulce, Com. & Sixto Camasca García & Astilla Blanca \\
\hline 13 & & Campesina San Julian Alto & Nery Pariona & Astilla Blanca \\
\hline 14 & \multirow{4}{*}{$\begin{array}{l}\text { Santiago de } \\
\text { Tucuma }\end{array}$} & Buenos Aires & Nicanol Cabana Castro & Almidón \\
\hline 15 & & Huayrapire & Moises Pérez Enciso & Astilla Blanca \\
\hline 16 & & Vuelo Pata & Reymundo Montañez & Chulpy \\
\hline 17 & & Usnupampa & Mario Oré Castro & Chulpy \\
\hline 18 & \multirow{2}{*}{ Pichos } & \multirow{2}{*}{ Muyupata } & Cirilo Yance Unsihuay & Pistiada \\
\hline 19 & & & Nora Aliaga Sedana & Carhuay \\
\hline 20 & \multirow{6}{*}{ Testigos } & INIA Cajamarca & Alicia Medina & Choclero-101 \\
\hline 21 & & INIA Cajamarca & Alicia Medina & $\begin{array}{c}\text { Choclero-INIA- } \\
603\end{array}$ \\
\hline 22 & & INIA Cajamarca & Alicia Medina & INIA-601 \\
\hline 23 & & Colección Local Cajamarca & Alicia Medina & Umutu \\
\hline 24 & & Colección Local Cajamarca & Alicia Medina & $\begin{array}{c}\text { Chaucha Semi } \\
\text { Blanco }\end{array}$ \\
\hline 25 & & Colección Local Cajamarca & Alicia Medina & Chaucha Rojo \\
\hline
\end{tabular}


En el área agronómica se hará énfasis en los aspectos agroecológicos, sobre todo en lo relacionado al manejo de las poblaciones de plantas (arreglos espaciales), manejo de las poblaciones de insectos plagas y de las principales enfermedades que afectan al cultivo en la región (Manejo integrado de plagas).

En lo que concierne a la producción de semilla, se establecerán lotes de producción de semilla en los campos de los agricultores, los cuales serán utilizados para implementar la metodología del mantenimiento de la identidad genética de las variedades de maíz. Esta metodología será dejada a los productores, quienes en adelante podrán iniciar sus propios programas de producción de semilla de alta calidad genética, física y fisiológica.

Se estima que esta acción estimule la producción de semilla de alta calidad, lo que contribuirá al desarrollo de la industria de semilla artesanal, un rubro que le dará valor agregado a los maíces amiláceos de la sierra peruana.

Una de las problemáticas detectadas con la recolección de las muestras de las variedades en la provincia, es su alto nivel de contaminación, lo cual confiere mayor heterogeneidad en las poblaciones, disminuyendo significativamente su potencial y la calidad del producto final. Por este motivo, para el tercer año del proyecto se establecerán los viveros para la purificación de estas variedades y la realización de cruzamientos controlados entre las diferentes poblaciones. Esta información permitirá estimar el nivel de heterosis entre las poblaciones y realizar los pool genéticos entre las variedades más relacionadas entre sí.

El uso de variedades más uniformes por los agricultores de la provincia, les brindará un valor agregado al producto cosechado, lo cual podrá mejorar los precios del maíz a nivel de los productores del campo.

Todo ello será posible gracias a la construcción de dos invernaderos que permitirán el establecimiento de viveros para la producción de semilla y para la realización de cruzamientos controlados, sin el temor de la presencia de heladas que puedan destruir los campos sembrados para estos propósitos.

Cabe mencionar la gran expectativa que ha generado en el seno de los productores de la provincia la ejecución de este proyecto de maíz, lo cual le está permitiendo a la UNAT proyectarse hacia las comunidades con resultados concretos y de gran relevancia para las organizaciones campesinas. De esta manera, los profesionales responsables de la propuesta de investigación en maíz trabajan arduamente para alcanzar los objetivos establecidos en el proyecto, que contribuirán significativamente al desarrollo integral de toda la provincia de Tayacaja, Huancavelica.

\section{REFERENCIAS BIBLIOGRÁFICAS}

Balzarini M.G., Gonzalez L., Tablada M., Casanoves F., Di Rienzo J.A., Robledo C.W. (2008). Manual 
del Usuario, Editorial Brujas, Córdoba, Argentina. Disponible en:https://www.researchgate.ne t/file.PostFileLoader.html?id=5 6d87439eeae395144018193\&a ssetKey=AS\%3A33560596059 3408\%401457026104675.

Banziger, M.; Barreto, H.J. 1999. Manual de usuario para fieldbook 5.1/7.1 y Alfa. México D. F. .CIMMYT. 48 p.

Hallauer A. 1985. Compendium of recurrent selection methods and their application. Critical Reviews in Plant Sciences. 3(1): 1-33.

Hugo Córdova. 1992. Respuesta diferenciales para rendimiento de híbridos de maíz evaluados en ambientes contrastantes de Latinoamérica, PCCMCA 1990 . A g r o no mía Mesoamericana, 3 (1): 1-8. D i s p o n ib 1 e e n : https://dialnet.unirioja.es/servle t/articulo?codigo $=5534240$.

Medina S., Marín C, Segovia V., Bejarano A., Venero Z., Ascanio R. y Meléndez E. 2002. Evaluación de la estabilidad del rendimiento de variedades de maíz en siete localidades de Venezuela. Agronomía Trop. 52 (3), 255-275. Disponible en: http://www.sian.inia.gob.ve/rev istas_ci/Agronomia\%20Tropic al/at5203/arti/medina_s.htm.

Paliwal, R. L. 2001a. Mejoramiento del maíz por selección recurrente. En: El maíz en los trópicos: mejoramiento y producción. Paliwal, R. L.; G. Granados; H. R. Laffite; A. D. Violic (Edes.). FAO, CIMMYT. Roma, 2001.

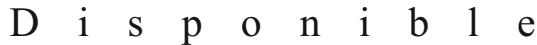
en:https://curlacavunah.files.w ordpress.com/2010/04/el-maizen-los-tropicos.pdf.

Paliwal, R. L. 2001b. Producción de semillas. En: El maíz en los trópicos: mejoramiento y producción. Paliwal, R. L.; G. Granados; H. R. Laffite; A. D. Violic (Edes.). FAO, CIMMYT. Roma, 2001. Disponible en: https://curlacavunah.files.word press.com/2010/04/el-maiz-enlos-tropicos.pdf.

Rosas J. C. 2001. Aplicación de metodologías participativas para el mejoramiento genético de frijol en Honduras. Agronomía Mesoamericana. 12 (2): 219-228. Disponible en: https://dialnet.unirioja.es/serv let/articulo?codigo=5039758.

Souza, A., G. Miranda, M. Gonzaga y L. Souza, L. 2009. Predicting the genetic gain in the Brazilian white maize landrace. Ciência Rural, v.39, p.19- 24, 2009. Dis pon íve I e m : http://www.scielo.br/pdf/cr/v 39n1/a04v39n1.pdf.

Toledo-Machado A., Arcanjo-Nunes J., Torres de Toledo Machado C., 
Lourenço- Nass L., Candido-da Rocha Bettero F. 2006. Mejoramiento participativo en maíz: su contribución en el empoderamiento comunitario en el municipio de Muqui, B rasil. Agronomía Mesoamericana 17(3): 393405 . Disponible en: https://www.alice.cnptia.embr apa.br/bitstream/doc/189286/ 1/ID291711.pdf.

\section{CORRESPONDENCIA}

Dr. Pedro García Mendoza

pejogam@gmail.com 